\title{
Cenplesto
}

\section{Acervos em rede: perspectivas para as instituições culturais em tempos de cultura digital}

\author{
Dalton Lopes Martins \\ Doutor; Universidade Federal de Goiás, Goiânia, GO, Brasil; \\ dmartins@gmail.com \\ Marcel Ferrante Silva \\ Doutor; Universidade Federal de Goiás, Goiânia, GO, Brasil; \\ marcelf@gmail.com \\ Danielle do Carmo \\ Mestre; Universidade Federal de Goiás, Goiânia, GO, Brasil; \\ docarmo.danielle@gmail.com
}

\begin{abstract}
Resumo: No contexto de transformações econômicas, culturais e sociais produzidas pelo desenvolvimento das tecnologias digitais e principalmente pelo surgimento e popularização da internet, a rede torna-se objeto paradigmático nas formas de comunicação e disseminação das informações. Sendo parte deste cenário, as instituições culturais têm produzido novas práticas e maneiras de lidar com as formas contemporâneas de armazenamento e disseminação das informações culturais a partir do objeto rede como elemento estruturante de seu fazer. O presente artigo tem como objetivo evidenciar as práticas de produção de acervos em rede, tanto em âmbito nacional quanto internacional. A análise dos dados coletados, por meio de pesquisa bibliográfica e documental, nos permitiu identificar iniciativas existentes no Brasil, assim como mapear práticas internacionais consolidadas em forma de serviços. Os resultados da pesquisa demonstram que enquanto as práticas de produção de acervos em rede encontram barreiras técnicas e políticas no cenário nacional, não tendo sido identificada nenhuma política de âmbito nacional em vigência, no cenário internacional essas práticas se mostram disseminadas e desenvolvidas na América do Norte, Europa e Oceania.
\end{abstract}

Palavras-chave: Acervos. Rede. Cultura digital. Instituições culturais.

\section{Introdução}

O fim do século XX e o início do século XXI foram marcados por profundas transformações econômicas e sociais, onde o desenvolvimento das tecnologias 
digitais e, em particular, pela emergência e popularização da internet, têm desempenhado um papel central junto a outros fatores influenciados e influenciadores dessas mudanças tecnológicas. Como elemento de pensamento e abstração organizacional que permite a reflexão de diversos movimentos em torno dessas transformações, encontra-se a ideia de rede como modo de representação da sociedade, elemento estruturante e dinamizador da circulação de fluxos comunicacionais e informacionais que acaba por atingir praticamente todas as áreas da sociedade e influenciar suas práticas e modos de funcionamento.

A rede torna-se objeto paradigmático e permite construir em torno de suas múltiplas possibilidades de definição novas formas de entender fenômenos sociais e, sobretudo, as práticas sociais e seus efeitos nos novos modos de se produzir cultura. Vale dizer que entende-se por prática social aquilo que se revela nas condutas regulares dos agentes em dinâmica de interação social, produzindo as coisas que se fazem e as coisas que não se fazem (THIRYCHERQUES, 2006). É importante considerar, portanto, que a rede torna-se uma chave de leitura fundamental dos movimentos sociais e culturais desde então, sendo que por meio dela é possível compreender as mudanças significativas nas condutas regulares dos agentes em relação, sua dinâmica e modo de ação.

A rede passa compor um novo modelo de desenvolvimento social e econômico:

[...] em que a aquisição, o armazenamento, processamento, valorização, transmissão, distribuição e disseminação de informação conducente à criação de conhecimento e à satisfação das necessidades dos cidadãos e das organizações, através das novas tecnologias da informação e comunicação, desempenham um papel central na atividade económica, na criação de riqueza, na definição da qualidade de vida dos cidadãos e das suas práticas culturais. (PINTO; SILVA, 2005, p. 2).

E sendo parte deste cenário, as instituições culturais têm produzido novas práticas e maneiras de se lidar com as formas contemporâneas de armazenamento e disseminação das informações culturais a partir do objeto rede como elemento estruturante de seu fazer. Dessa forma, as instituições deparam- 
se com o desafio de adotar as tecnologias digitais em seus processos internos de gestão e na relação com seus usuários e, sobretudo, na potencialização de ações e projetos derivados da tríade preservação, pesquisa e comunicação.

Atualmente, a criação e manutenção de sites institucionais parecem constituir parte importante da prática comunicacional das instituições culturais, tanto na divulgação de informações e ações quanto na difusão de acervos digitalizados.

Como resultado, têm-se as várias exposições virtuais ou ciber exposições (domínio no qual o museu pode apresentar uma expertise real), os catálogos digitalizados, os fóruns de discussão mais ou menos sofisticados, e as diversas incursões dessas instituições nas redes sociais [...]. (DESVALLÉES; MAIRESSE, 2013, p. 37).

Nesse contexto, uma questão específica nos chama atenção: as práticas produzidas pelas instituições culturais em torno de suas estratégias de criação de acervos digitais e o modo como esse processo tem sido pensado quanto à socialização, apropriação e circulação da informação em rede. Tradicionalmente, os acervos culturais digitalizados são tratados a partir de uma prática de organização da informação que tem como preocupação central o armazenamento, a organização, a rotulagem, a preservação e a distribuição de seus conteúdos por parte de uma instituição. O que se observa é que essa prática é uma das que vêm se modificando de maneira profunda desde o final dos anos 90 ao incorporar o elemento rede como paradigma de organização e integração dos acervos digitalizados, revelando novas práticas bastante características das maneiras próprias de exercer a cultura digital por parte das instituições culturais. É objetivo deste artigo, portanto, evidenciar práticas de produção de acervos culturais em rede, tanto em âmbito nacional quanto internacional, identificando e buscando realizar um diagnóstico das práticas nacionais, também buscamos mapear e entender como operam as práticas já consolidadas como serviços no cenário internacional. Entende-se que ao mapear essas práticas e evidenciar possíveis diferenças em seus modos de funcionamento, pode-se contribuir para a 
reflexão sobre a constituição de uma política nacional para acervos digitais em rede.

\section{Procedimentos metodológicos}

O presente trabalho é resultado de um processo investigativo de abordagem qualitativa e natureza exploratória. Para a realização deste trabalho, optou-se pela pesquisa bibliográfica e documental. Para a coleta de dados, foram realizadas buscas tanto em bases especializadas em literatura científica, como a Base de Dados Referenciais de Artigos e Periódicos em Ciência da Informação (BRAPCI) e o Google Acadêmico, quanto em ferramentas de buscas de dados na internet como o Google. As buscas utilizaram como descritores as seguintes palavras-chave: "acervos digitais", "repositórios institucionais", "acervos em rede", "serviços de informações culturais" e variações das mesmas na língua inglesa e portuguesa, considerando plural e singular. Os resultados encontrados foram levados em consideração para a pesquisa apenas quando se referiam à temática central do trabalho, qual seja a dos acervos em rede na perspectiva das políticas culturais. Dessa forma, foi possível encontrar documentos, sites, pesquisas e trabalhos científicos que nos permitiram identificar e diagnosticar o cenário brasileiro relativo às práticas de produção de acervos culturais em rede, assim como alguns serviços dessa natureza que operam no cenário internacional.

\section{Da produção do problema no Brasil: indícios sociais que apontam a necessidade dos acervos em rede}

Segundo a Carta de Recife 2.0, instituições culturais responsáveis pela preservação e acesso dos acervos culturais têm desenvolvido diversas iniciativas de digitalização e publicação de seus acervos, mas observa que essas iniciativas se apresentam de forma desconexa (REDE MEMORIAL DE PERNAMBUCO, [2012]). A ausência de conexão entre essas iniciativas acaba por enfraquecer o potencial de integração e disseminação de seus acervos digitais. Para agregar esses conteúdos de forma acessível, faz-se necessário a concepção de uma 
política integrada que procure dar condições de visibilidade e aplicabilidade a estratégias técnicas, políticas, culturais e econômicas que facilitem a produção de uma rede de acervos que, dialogando a mesma língua a partir da adoção de padrões compartilhados, podem se interconectar e se tornarem acessíveis de forma integrada.

Outra iniciativa que busca evidenciar o problema no país é o documento intitulado Carta de Ribeirão Preto, elaborado em 2015, na ocasião do Workshop de Preservação Digital, realizado no Centro de Tecnologia da Informação da Universidade de São Paulo e promovido pela Seção da América Latina e Caribe da Federação Internacional de Associações de Biblioteca e Instituições. O documento, assinado por representantes de vinte quatro instituições, incluindo membros da Rede Memorial, manifesta inquietações relativas à necessidade da formalização de uma Política Nacional de Acervos Digitais, tendo em vista a pulverização de iniciativas governamentais e institucionais que tem atuado "[...] em distintas frentes duplicando esforço e diminuindo a eficiência do gasto público [...]” (REDE MEMORIAL DE PERNAMBUCO, 2015, doc. não paginado).

O debate em território brasileiro em torno da questão tem se intensificado de quase uma década para cá, onde inspirado pelos debates realizados no Simpósio Internacional de Políticas Públicas para Acervos Digitais, realizado pelo Ministério da Cultura (MinC), em São Paulo, em abril de 2010, o documento de mesmo nome apresenta propostas ao MinC e aos agentes do setor com o intuito de delinear um Plano Nacional de Digitalização e Acesso à Cultura e Conhecimento. Entre as propostas encontramos a seguinte:

[...] criação de uma plataforma que organize a troca de informações entre as instituições e também ofereça possibilidades múltiplas de acesso aos conteúdos armazenados em formato digital dentro das instituições públicas ou privadas que forem abarcadas pelo Plano Nacional de Digitalização e Acesso a Cultura e Conhecimento. (TADDEI, 2010, p. 6).

Elementos debatidos no seminário apontam que essa plataforma teria como objetivo a gestão e disponibilização em rede dos conteúdos digitalizados 
pelas entidades brasileiras de cultura, informação, história e conhecimento. Apesar de existirem alguns projetos nesse sentido, ainda não é possível encontrar, em pleno funcionamento, um plano nacional que integre de fato a base de dados de diversas instituições culturais do território nacional e sequer proponha padrões mínimos de adoção que deveriam ser seguidos para que tal objetivo pudesse ser alcançado. Sabe-se que a ideia era a produção de um banco de dados de acervos culturais acessível por meio de um mecanismo de busca único que potencializaria de forma significativa as iniciativas de digitalização e disponibilização de acervos em rede.

A ausência de uma política que realize essa integração acaba por dificultar processos de mapeamento e diagnóstico acerca das condições dos objetos culturais, além de limitar as possibilidades de cruzamento de dados e produção de indicadores que facilitem o monitoramento dos efeitos das políticas específicas para o setor. Como se observa, o problema vai além da questão da disponibilização e socialização da informação em rede, mas incide também em aspectos fundamentais de gestão da política cultural, onde a demanda pela capacidade de diagnosticar setores, áreas de atuação e se conhecer o que de fato existe torna-se possível apenas pela integração da informação a partir de protocolos padronizados que permitem cruzamentos e sínteses de métricas e indicadores.

Para promover a integração entre bancos de dados de acervos de diferentes instituições culturais e colocá-los em rede se torna necessário que o princípio da interoperabilidade seja observado. A interoperabilidade nada mais é que "[...] um conjunto de mecanismos de mediação que torna possível a comunicação entre diferentes sistemas, sendo eles informatizados ou não." (OLIVEIRA; MACULAN; GOMES, 2016, p. 7). Uma das dificuldades de interoperar acervos é o uso de diferentes softwares de gestão:

Arquivos, bibliotecas e museus disponibilizam seus catálogos e/ou coleções digitais na Internet, tornando-os acessíveis a qualquer usuário, em qualquer lugar, a qualquer momento. Há, no entanto, grande dificuldade em tornar interoperáveis acervos hospedados em diferentes instituições, uma vez que esses acervos são dependentes de softwares específicos para publicar seus registros na Web. Os 
atuais softwares de gestão de catálogos - usados em arquivos, bibliotecas e museus -, também não permitem que sejam feitos links para um registro específico, excluindo seus registros de toda a economia da Web, com base na publicação de conteúdos e de links entre eles. (SANTOS NETO et al., 2013, p. 81).

Outra grande dificuldade é a característica heterogênea dos objetos digitais que compõem os bancos de dados de diferentes instituições culturais. Dessa forma Marcondes (2016, p. 66), aponta que:

[...] as experiências de interoperabilidade mais consistentes têm origem na área de bibliotecas, onde os acervos são bastante homogêneos. Quando se trata de integrar acervos de arquivos, bibliotecas e museus, ao contrário, a heterogeneidade dos acervos é bastante significativa.

No processo de criação de repositórios para armazenar acervos digitalizados, as instituições acabam por adotar sistemas e metodologias específicas, o que acaba por tornar seus catálogos isolados, fechados em si mesmo.

Durante anos essas instituições têm desenvolvido semânticas, modelos, formatos e padrões próprios. Todo o potencial de integração e acesso proporcionado pela Web não pode ser aproveitado pelos sistemas convencionais que gerenciam esses catálogos, impedindo que tais acervos possam aproveitar suas sinergias e potenciais culturais, e que se beneficiem, e também que outras instituições possam fazer links para seus acervos. (MARCONDES, 2012, p. 181).

Os custos de implementação e manutenção da infraestrutura tecnológica, assim como a necessidade de mão de obra especializada se apresentam como barreiras no processo de digitalização, armazenamento e socialização de acervos culturais. As dificuldades e desafios identificados são desdobramentos de um problema central que se configura no fato de ainda não existir uma política nacional específica que estabeleça procedimentos, padrões e parâmetros técnicos que possibilitem a realização de ações coordenadas e colaborativas que possuam como objetivo comum, a digitalização e a disponibilização de acervos culturais em rede por meio de banco de dados integrado e plataformas interoperáveis. 
A situação brasileira e, ao mesmo tempo, o potencial brasileiro para a produção de acervos digitais pode ser entendido mais de perto quando observados os dados fornecidos por importantes pesquisas realizadas e mecanismos de gestão adotados pelo Instituto Brasileiro de Museus (IBRAM). O Cadastro Nacional de Museus referente ao ano de 2015, realizado pelo IBRAM, verificou a existência de 3.619 museus no território brasileiro entre públicos e privados, destes, 2.010 museus declararam possuir algum tipo de acervo. No ano de 2014, a Pesquisa Anual de Museus, também realizada pelo IBRAM, indica a existência de 56.871 .061 bens culturais em acervos em uma amostra de 931 museus que participaram da pesquisa e declararam possuir bens culturais em seus acervos (INSTITUTO BRASILEIRO DE MUSEUS, 2014; 2015). Com base nos resultados das duas pesquisas citadas anteriormente, é possível inferir que as instituições museais brasileiras possuem em seus acervos aproximadamente mais de 122 milhões itens. Quanto aos meios de registro e inventário, 84,9\% museus que declararam na PAM 2014 que estão em processo de realização de inventário ou registro e 66,0\% utilizam algum sistema informatizado para os registros dos bens culturais (INSTITUTO BRASILEIRO DE MUSEUS, 2014).

Como se observa, os dados revelam um cenário de enorme potencial em termos de objetos passíveis de digitalização e a produção de bases de dados de enorme riqueza cultural e de grande potencial de interesse público, graças à diversidade e qualidade da produção cultural brasileira. No entanto, há ainda em torno de $40 \%$ das instituições que carecem de informatização, com a disponibilidade de sistemas de gestão para registro dos bens culturais e, eventualmente, disponibilização dos acervos em rede. Para além dessas, há as $66 \%$ que já possuem sistema informatizado onde é necessário se compreender que sistemas são esses, que usos são feitos e que potencial de interoperabilidade e disponibilidade dos acervos em rede existe de fato nessas instituições e nos sistemas que utilizam.

Não é possível saber com exatidão quantos acervos e a quantidade de bens culturais que estão digitalizados ou se encontram em processo de 
digitalização. O Plano Nacional de Cultura (PNC), instituído pela Lei nº 12.343, de 2 de dezembro de 2010 (BRASIL, 2010), prevê que, até 2020, estejam disponíveis na internet todas as obras audiovisuais da Cinemateca Brasileira e do Centro Técnico Audiovisual (CTAv); todo o acervo da Fundação Casa de Rui Barbosa; todos os inventários e ações de reconhecimento realizados pelo Instituto do Patrimônio Histórico e Artístico Nacional (IPHAN); todas as obras de autores brasileiros do acervo da Fundação Biblioteca Nacional (FBN) e todo o acervo iconográfico, sonoro e audiovisual do Centro de Documentação da Fundação Nacional das Artes (Cedoc/Funarte). Além disso, 100\% das bibliotecas públicas e $70 \%$ dos museus e arquivos deverão disponibilizar informações sobre seus acervos no Sistema Nacional de Informações e Indicadores Culturais (BRASIL, 2011). O histórico da meta apresenta dados referentes à digitalização de acervos de seis ${ }^{1}$ instituições brasileiras e concluí que, em 2015, a média do percentual de acervos disponibilizados na internet foi apenas de 8\% (BRASIL, 2017).

Quanto aos projetos que promovem a integração de acervos digitalizados no Brasil, a presente pesquisa identificou três iniciativas que buscam desenvolver ações na direção da integração de acervos em rede. São elas: a Rede Web de Museus/Rede MUSA, do estado do Rio de Janeiro (2017), de alcance estadual, e de alcance nacional, podemos citar a Rede Memória da Fundação Biblioteca Nacional e a Rede Memorial de iniciativa da sociedade civil organizada.

A Rede Web de Museus é uma iniciativa da Secretaria de Cultura do Estado do Rio de Janeiro e integra acervos digitalizados de treze ${ }^{2}$ instituições culturais do estado. O sistema se encontra disponível na internet e por meio da ferramenta de busca é possível realizar consultas ao banco de dados das diferentes instituições participantes. A plataforma permite a visualização das mídias das obras e é possível obter informação sobre os itens do acervo. Iniciativa da Fundação Biblioteca Nacional, a Rede Memória Virtual Brasileira tem entre seus objetivos apoiar a disponibilização, por meio eletrônico, dos acervos das bibliotecas parceiras com o intuito de inventariar e disseminar o 
conteúdo cultural brasileiro que se encontra armazenado em coleções pelo país. Sua plataforma encontra-se acessível online e apresenta possibilidade de busca em acervos de dezesseis instituições culturais.

Outra iniciativa de abrangência nacional é a Rede Memorial, nascida no âmbito das discussões da Rede Memorial de Pernambuco, tem como objetivo a articulação nacional das instituições comprometidas com políticas de digitalização de acervos memoriais. Essa rede se baseia nos princípios da Carta de Recife 1.0, que foi redigida em ocasião da primeira reunião da rede, realizada em 2011. Apesar das articulações institucionais, a Rede Memorial ainda não possui uma plataforma com banco de dados de acervos disponíveis para consulta online.

Embora atualmente no Brasil existam algumas iniciativas que objetivam conectar os acervos de instituições culturais e disponibilizá-los em rede, o objetivo de alcançar a abrangência do território brasileiro ainda se encontra longe de ser alcançado. No entanto, tanto pesquisadores e acadêmicos da área, ações governamentais em âmbito federal e estadual quanto ações da sociedade civil organizada vêm demonstrando a preocupação com a questão e produzindo esforços no sentido de enunciar a necessidade de uma política que procure incentivar a produção de acervos em rede no país. As práticas encontradas ainda são muito limitadas, isoladas e carecem de apoio técnico e de modelos de governança escaláveis para o território brasileiro. O que se identifica hoje no país é uma falta de incentivo explícito, tanto político quanto econômico, que especificamente procure ofertar soluções para a questão dos acervos e articular as instituições culturais nessa direção.

\section{Experiências internacionais: os acervos em rede como valor social}

O relatório intitulado Europeana as online cultural information service, referente a setembro de 2016, apresenta um panorama mundial e faz uma análise de 20 serviços de acervos em rede que tem como objetivo a distribuição de algum tipo de conteúdo cultural, são eles: Europeana, Archives Portal Europe APE, ARTStore,British Library, Digital Public Library of America - DPLA, 
DigitalNZ, Google, Google Art's \& Culture, Hathi Trust, Internet Archive, JStore, The European Library -TEL, Trove, Wikipédia, Wikimedia Commons, World Digital Library, Smithsonian, RijkdMuseum, EUScreen e Galica (NAVARRETE, 2016).

Os serviços de conteúdo cultural analisados apresentam uma diversidade de tipos de objetos digitais que são disponibilizados, tamanho de acervo, modelo de governança, forma de financiamento e o tipo de serviço que oferecem, conforme a Tabela 1 .

Tabela 1 - Panorama dos serviços

\begin{tabular}{|c|c|c|c|c|c|c|}
\hline \# & Serviço & Serviço Primário & $\begin{array}{l}\text { Tamanho da } \\
\text { coleçäo Online }\end{array}$ & Financiamento & $\begin{array}{c}\text { Data de } \\
\text { Lançamento } \\
\text { digital }\end{array}$ & Governança \\
\hline 1 & Europeana & Agregador de índice & 53000000 & Público & 2008 & Rede \\
\hline 2 & APE & Agregador de índice & 249000000 & Público & 2009 & Rede \\
\hline 3 & ARTster & Biblioteca Digital & 2000000 & Privado & 2001 & ONG \\
\hline 4 & British Library & Biblioteca Digital & 13000000 & Público & 1994 & ONG \\
\hline 5 & DPLA & Agregador de indice & 2000000 & Público & 2013 & ONG \\
\hline 6 & DigitalNZ & Agregador de índice & 29000000 & Público & 2006 & Rede \\
\hline 7 & Google & Agregador de índice & - & Privado & 1998 & Empresa \\
\hline 8 & Google Art & Agregador de exibição & 6000000 & Privado & 2011 & Empresa \\
\hline 9 & Hathi Trust & Biblioteca Digital & 29000000 & Universidade & 2006 & ONG \\
\hline 10 & Internet Archive & Agregador de índice & 4300000 & Público/Privado & 1996 & ONG \\
\hline 11 & JSTORE & Biblioteca Digital & 8000000 & Público/Privado & 1997 & ONG \\
\hline 12 & TEL & Biblioteca Digital & 200000000 & Público & 2005 & Rede \\
\hline 13 & Trove & Agregador de exibição & 491000000 & Público & 2008 & Rede \\
\hline 14 & Wikipédia & Enciclopédia & 32200000 & Público & 2001 & ONG \\
\hline 15 & Wikimedia Commons & Biblioteca de mídia & 39500000 & Público & 2004 & ONG \\
\hline 16 & World Digital Library & Biblioteca Digital & 14000 & Público/Private & 2009 & ONG \\
\hline 17 & Smithsonian & Biblioteca Digital & 9600000 & Público/Private & 1985 & ONG \\
\hline 18 & Rijikdmuseum & Biblioteca Digital & 1000000 & Público & 2002 & Museu \\
\hline 19 & EUScreen & Agregador de indice & 1060000 & Público & 2011 & Rede \\
\hline 20 & Galica & Biblioteca Digital & 1600000 & Público & 1997 & ONG \\
\hline
\end{tabular}

Fonte: Adaptado de Navarrete (2016, tradução nossa).

A Europeana é uma plataforma voltada para o patrimônio cultural europeu, fundada pela European Commission, que agrega e disponibiliza online conteúdos culturais de diversas instituições da União Europeia. O Archives Portal Europe - APE é um portal gerido pela Archivel Portal Europe Foundation que reúne e disponibiliza informações e documentos digitais de diversos países da Europa. O ARTStore é uma biblioteca digital que armazena imagens e mídias digitais de diversos museus, arquivos, artistas e instituições 
educacionais de várias partes do mundo, e é gerida pela organização não governamental ITHAKA. A British Library é a biblioteca nacional do Reino Unido que armazena conteúdo cultural analógico e digital dos países do Reino Unido. A Digital Public Library of America - DPLA é uma plataforma estadunidense que reúne conteúdos culturais digitais de livrarias, arquivos e museus de todo o país, e é mantida por diversas fundações além de agências governamentais. $O$ DigitalNZ é um portal gerido pela Biblioteca Nacional da Nova Zelândia que reúne acervos de diferentes instituições culturais do país. $\mathrm{O}$ Google é uma empresa de serviços online e software dos Estados Unidos que disponibiliza, por meio de sua ferramenta de busca, conteúdos culturais disponíveis na internet. O Google Art's \& Culture é uma biblioteca de mídia que reúne acervos culturais digitais de diversas instituições ao redor do mundo, e é gerida pelo Google Cultural Institute. O HathiTrust é uma biblioteca digital estadunidense que armazena conteúdos culturais de diferentes instituições do país, o projeto é liderado pela University of Michigan, Indiana University, University of Virginia, e University of California. O Internet Archive é um portal que reúne conteúdos culturais digitais tanto de origem institucional quanto individual, e é gerido por uma organização sem fins lucrativos de mesmo nome. O JStore é uma biblioteca digital gerida por uma organização não governamental chamada ITHAKA, e tem como objetivo reunir trabalhos acadêmicos, livros e fontes primárias. A The European Library -TEL é um portal online não-comercial que dá acesso a coleções de quarenta e oito bibliotecas nacionais da Europa. O Trove é uma plataforma da Biblioteca Nacional da Austrália que agrega e disponibiliza conteúdos culturais digitais de bibliotecas, museus, arquivos, repositórios e etc. A Wikipédia é um projeto de enciclopédia colaborativa, que tem como propósito fornecer conteúdos culturais de acesso livre, é gerida pela entidade sem fins lucrativos Fundação Wikimedia. A Wikimedia Commons é um repositório de mídia que reúne conteúdos culturais de diversas partes do mundo, é um projeto sem fins lucrativos gerido também pela Fundação Wikimedia. A World Digital Library é uma biblioteca digital que disponibiliza conteúdo cultural de diversos países e culturas ao redor do mundo, é um projeto gerido por uma organização não governamental, possuí 
colaboração da Biblioteca do Congresso dos EUA e da Organização das Nações Unidas para a Educação, a Ciência e a Cultura (UNESCO). O Smithsonian é uma instituição museal norte americana composta por um complexo de dezenove museus, é gerida por uma organização não governamental e disponibiliza parte de seu acervo digital para consulta online. O RijkdMuseum, o Museu Nacional Holandês, disponibiliza seu acervo digitalizado para consulta por meio de sua plataforma online. O EUScreen é um portal online europeu que oferece acesso livre a milhares de conteúdos culturais digitalizados. A Galica é a biblioteca digital da Biblioteca Nacional da França, e oferece acesso a milhares de conteúdos culturais digitalizados de seu acervo e acervos parceiros, e é gerida por uma organização não governamental.

Os conteúdos culturais disponibilizados pelos serviços ARTStor, British Library, DPLA, Google Art's \& Culture, Hathi Trust, JSTORE, TEL, World Digital Library, Smithsonian, RijkdMuseum, EUScreen e Galica têm como origem somente instituições guardiãs de acervos culturais. Já os conteúdos disponibilizados pelos serviços Trove, Wikimedia Commons e Wikipédia, tem origem institucional, individual e automatizada. O serviço Google disponibiliza conteúdo de origem automatizado por meio de sua ferramenta de busca. O serviço Europeana disponibiliza conteúdos de origem individual e institucional, e o Internet Archive possui conteúdos de origem individual e automatizado.

Os serviços baseados no modelo de digital library e media library, possuem um banco de dados próprio e a ferramenta de busca fica restrita a esse banco de dados. Já os baseados em aggregated index e aggregated exhibition são aqueles cujos resultados das buscas estão armazenados em diferentes instituições e não em um banco de dados próprio do serviço. Entre os serviços digital library, os que possuem uma maior coleção em seu banco de dados são o The European Library - TEL com 200 milhões de itens online, seguido pelo HathiTrust, com 29 milhões, e pela British Library, com 13 milhões de itens digitalizados online. Entre os serviços que possuem mais itens agregados no resultado de sua ferramenta de buscas são o Trove, com 491 milhões de itens, 
seguido pelo o APE, com 249 milhões, e pela Europeana, com 53 milhões de itens agregados em sua busca.

Quanto ao tipo de acervo disponibilizado, a maioria deles possui em suas coleções imagens, textos e conteúdo audiovisual e apenas uma pequena parcela deles possui objetos digitalizados em 3D, websites e softwares como podemos observar na imagem.

Figura 1- Tipo de objeto por serviço

\section{Object Type in Collection}

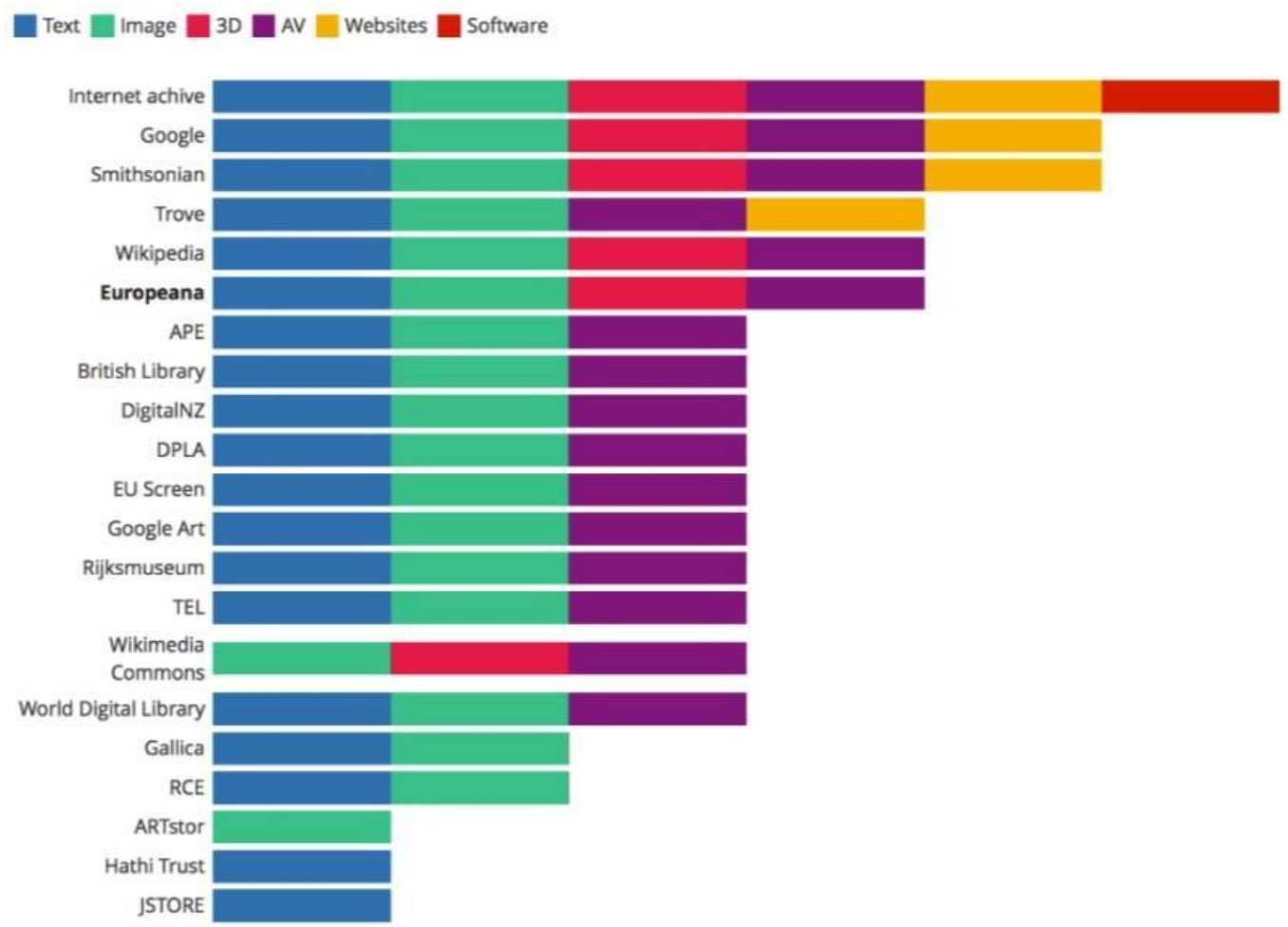

Fonte: Navarrete (2016).

Em relação à abrangência geográfica, podemos observar que o serviço Trove abrange somente conteúdos culturais relativos à Austrália, tendo sua área de abrangência limitada por apenas uma região, assim como DigitalNZ que abrange somente a Nova Zelândia. Entre aqueles que têm maior alcance está o Google Art's \& Culture, que abrange parte dos Estados Unidos, da América Central, da América do Sul, da Europa, da Ásia e parte da Oceania. A World 
Digital Library abrange o continente americano, o continente africano, o continente europeu e parte do continente asiático, já a Europeana apresenta conteúdos culturais online que abrangem todo o continente europeu.

Figura 2 - Cobertura geográfica por serviço

\section{Geographical coverage of cultural information services}

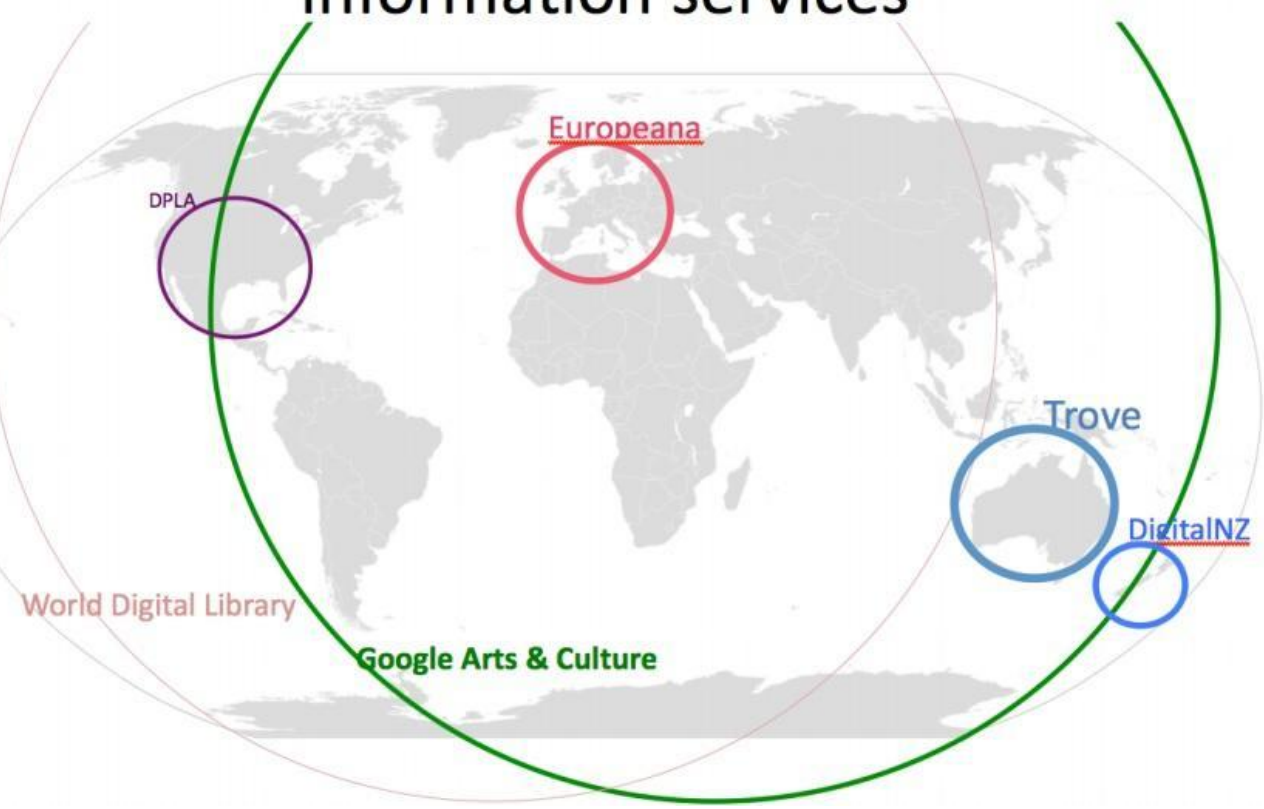

Fonte: Navarrete (2016).

Como podemos observar na imagem acima, os únicos serviços de conteúdo cultural online que abrangem o território brasileiro são a World Digital Library e o Google Art's \& Culture. A World Digital Library é um serviço que agrega aproximadamente 14 mil itens digitais de diversas instituições, esse serviço é gerido por uma organização não governamental e tem financiamento misto, recursos públicos e privados. O Google Art's \& Culture possui registros de aproximadamente 60 milhões de itens em seus registros, é gerido por uma empresa particular e tem financiamento privado.

Há dois serviços dos 20 apresentados acima que realizaram pesquisa específica sobre o impacto econômico de seus acervos para os territórios onde atuam. É importante ressaltar que tal tipo de estudo visa demonstrar de forma 
objetiva os impactos sociais desse tipo de estratégia de integração de acervos, tornando mais fácil de compreender que valores intangíveis e imateriais, tais como a produção cultural de um país, tornam-se uma riqueza não apenas de forma subjetiva, mas também impactando na produção de riqueza material economicamente mensurável. A British Library, também conhecida como Biblioteca Nacional do Reino Unido, reúne um acervo digitalizado de aproximadamente 13 milhões de itens. O estudo econômico produzido pela Oxford Economics (TESSLER, 2013), publicado em janeiro de 2013, apontou que os serviços online da British Library estão avaliados em 19.5 milhões de libras por ano, gerando benefícios para estudantes, pesquisadores e cidadãos do Reino Unido. O estudo intitulado The Value of Europeana, publicado em 2013, pela SEO Economic Research, calculou o impacto da Europeana na economia da União Europeia e identificou cinco grupos beneficiados pelos serviços oferecidos. O primeiro grupo é composto por instituições culturais, o segundo pelo público em geral, o terceiro grupo seria composto pela indústria do turismo, o quarto grupo indústrias criativas, e o quinto grupo são os pesquisadores e instituições de ensino. Ao fazer a análise da importância econômica dos serviços da Europeana, chegou-se à conclusão de que o impacto econômico para o continente europeu seria de aproximadamente 78.8 milhões de euros em um cenário de base, 59.6 milhões de euros em um cenário pessimista e 97.7 milhões em um cenário otimista (POORT et al., 2013).

Os resultados acima apresentados demonstram práticas bastante diferentes das encontradas no cenário nacional. Inicialmente, é possível notar que as principais regiões mais desenvolvidas do mundo possuem políticas locais para a produção de acervos em rede, onde encontramos ações específicas na Oceania, na América do Norte, com foco específico nos Estados Unidos da América, e na Europa. O tamanho dos acervos é altamente expressivo, sendo o menor deles da ordem de 2 milhões de objetos, no caso da DPLA. Há grande diversidade dos tipos de acervos disponibilizados, sendo que a maioria dos serviços oferta texto, imagem e conteúdos audiovisuais. Vários deles possuem modelo de governança baseado em rede, indicando que diferentes instituições possuem espaço de participação e operam a partir de parâmetros compartilhados 
e acordados para viabilizar seu funcionamento integrado. O impacto econômico dos serviços que se propuseram a realizar esse tipo de análise demonstra resultados surpreendentes, aponta impactos expressivos e, como no caso da Europeana, chega a superar os valores investidos na manutenção do serviço, funcionando como um elemento que não apenas realiza um importante e fundamental papel cultural, mas também desempenha ação de ativação da economia da União Europeia. As práticas aqui encontradas demonstram alta capacidade de articulação em rede, a superação das dificuldades de integração de acervos oriundos de fontes e mídias distintas, aproveitando de forma convergente com a cultura digital vigente dos efeitos de rede, agregando instituições e objetos culturais digitalizados em padrões compartilhados, facilitando e estimulando a circulação de informação estratégica de sua produção cultural, valorizando socialmente e economicamente sua cultura.

\section{Conclusão}

A Declaração Universal de Direitos Humanos estabelece, no artigo 19, que todo indivíduo possui o direito fundamental de "[...] procurar, receber e transmitir informações e ideias por quaisquer meios e independentemente de fronteiras." (ORGANIZAÇÃO DAS NAÇÕES UNIDAS PARA A EDUCAÇÃO, A CIÊNCIA E A CULTURA, 1998, doc. não paginado). Esse artigo, escrito em 1948, toma novas proporções na era da informação, pois se no passado, o acesso à informação estava limitada por barreiras territoriais, econômicas e linguísticas, hoje com a internet e sua popularização, o direito ao acesso à informação não somente é desejável mas totalmente realizável pelo meio digital. Somado a isso, a Declaração Universal de Direitos Humanos também estabelece, no artigo 22, que os direitos culturais, entre outros, são indispensáveis à dignidade humana. Assim como "[...] participar livremente da vida cultural da comunidade, de fruir as artes e de participar do processo científico e de seus benefícios." (ORGANIZAÇÃO DAS NAÇÕES UNIDAS PARA A EDUCAÇÃO, A CIÊNCIA E A CULTURA, 1998, doc. não paginado). 
A Declaração de Vancouver, intitulada A memória do Mundo na Era Digital, diz que “[...] a informação digital está sendo perdida porque seu valor é subestimado seja por causa da ausência de sistemas legais ou institucionais, seja porque faltam conhecimento, habilidade e/ou fomento para os que têm a custódia do acervo." (ORGANIZAÇÃO DAS NAÇÕES UNIDAS PARA A EDUCAÇÃO, A CIÊNCIA E A CULTURA, 2012, doc. não paginado). Dessa forma a UNESCO, desde o início do século XXI, recomenda que seus estados membros promovam a cooperação entre o legislativo e as instituições culturais guardiãs de acervos culturais de forma a estabelecer leis e políticas públicas que viabilizem e apoiem a preservação do patrimônio digital e o acesso ao cidadão, garantindo a formulação de padrões e linhas mestras a serem adotadas nos processos de preservação digital.

A presente pesquisa procurou contextualizar a produção de acervos em rede como uma nova prática cultural que surge no desdobrar de uma sociedade contemporânea que se descobre em rede. A rede, como paradigma organizacional, orienta o olhar para diversos fenômenos sociais em acontecimento nos tempos atuais, permitindo compreender novos fenômenos relacionais que produzem novas práticas. Na cultura, certamente, isso não seria diferente. Os resultados da pesquisa demonstram, no contexto brasileiro, uma grande sensibilização à necessidade de produção de acervos em rede na área cultural, demonstrando que a prática não se instaura como processo de gestão e produção da área por um conjunto de limitações que ainda não puderam ser superadas, tanto do ponto de vista técnico como político. Ainda não se produziu, no país, um processo político com força suficiente para incidir em uma ampla articulação nacional que crie como solução um modelo de governança que garanta a participação dos diversos atores sociais e instâncias públicas e privadas. Já no cenário internacional, a situação se apresenta de outra maneira. A prática de produção de acervos em rede se encontra disseminada nas regiões mais desenvolvidas economicamente, apresentando soluções locais com diversos modelos de governança e características próprias. Algumas demonstram, de forma objetiva, os impactos econômicos gerados, levando a crer 
que ações nessa direção podem também servir como elementos indutores de ativação da economia e inovação.

No Brasil, segundo a Constituição de 1988, nos artigos 23 e 215, é competência comum da federação, estados e municípios “[...] proporcionar meios de acesso à cultura [...]", assim como garantir a todos “[...] o pleno exercício dos direitos culturais e acesso às fontes da cultura nacional [...]" (BRASIL, 1988, doc. não paginado). Considerando o contexto econômico atual, e os desafios do setor cultural, as metas colocadas pelo Plano Nacional de Cultura parecem ser de difícil alcance. Dessa forma, torna-se imperativo o desenho de uma política nacional que promova a busca por soluções que auxiliem na construção de perspectivas de interoperabilidade entre os diferentes acervos. Para isso, torna-se necessário a retomada do protagonismo político nesse processo, somente dessa forma será possível que o trabalho de digitalização dos acervos culturais brasileiros seja executado pelas instituições culturais de forma orientada e coordenada, integrando as informações e facilitando o acesso à população brasileira. Para essa finalidade, também tornase necessário que o poder público se posicione e articule o desenho de um modelo de governança capaz de direcionar, monitorar, supervisionar e avaliar as iniciativas de digitalização, armazenamento e disponibilização de acervos em rede, preferencialmente por meio de um modelo de gestão que envolva o Estado e a sociedade civil de forma participativa, transparente e democrática.

As experiências internacionais podem servir de subsídio para o desenho desse modelo de governança e também para a adoção de tecnologias, plataformas e protocolos de interesse comum. Espera-se, como trabalho futuro desta pesquisa, a avaliação e comparação detalhada dos modelos de governança das iniciativas internacionais apresentadas. Entende-se que dessa avaliação será possível apontar de forma mais objetiva características e modos de ação que podem favorecer e fortalecer o desenvolvimento dessa prática cultural no país, trazendo benefícios sociais e econômicos significativos para o Brasil. 


\section{Referências}

BRASIL. Constituição (1988). Constituição da República Federativa do Brasil. Brasília, 1988.

BRASIL. Lei $\mathrm{n}^{\circ}$ 12.343, de 2 de dezembro de 2010. Institui o Plano Nacional de Cultura - PNC, cria o Sistema Nacional de Informações e Indicadores Culturais SNIIC e dá outras providências. Diário Oficial [da] União, Brasília, 3 dez. 2010 .

BRASIL. Ministério da Cultura. Metas do Plano Nacional de Cultura. 2011.

DESVALLÉES, André; MAIRESSE, François (Ed.). Conceitos-chave de museologia. São Paulo: Armand Colin, 2013.

INSTITUTO BRASILEIRO DE MUSEUS. Cadastro nacional de museus. Brasília, 2015.

INSTITUTO BRASILEIRO DE MUSEUS. Pesquisa anual de museus 2014. Brasília, 2014.

MARCONDES, Carlos Henrique. Interoperabilidade entre acervos digitais de arquivos, bibliotecas e museus: potencialidades das tecnologias de dados abertos interligados. Perspectivas em Ciência da Informação, Belo Horizonte, v. 21, n. 2, p. 61-83, abr./jun. 2016.

MARCONDES, Carlos Henrique. "Linked data" - dados interligados - e interoperabilidade entre arquivos, bibliotecas e museus na web. Encontros Bibli, Florianópolis, v. 17, n. 34, p. 171-192, maio./ago. 2012.

NAVARRETE, Trilce. Europeana as online cultural information service: study report. [S.1.]: Europeana, 2016.

OLIVEIRA, Dalgiza Andrade; MACULAN, Benildes C. M. S.; GOMES, Marcos Aurélio. Equipamentos culturais e políticas públicas: a interoperabilidade em questão. Ciência da Informação em Revista, Maceió, v. 3, n. 1, p. 3-10, jan./abr. 2016.

ORGANIZAÇÃO DAS NAÇÕES UNIDAS PARA A EDUCAÇÃO, A CIÊNCIA E A CULTURA. Declaração UNESCO/UBC Vancouver. 2012.

ORGANIZAÇÃO DAS NAÇÕES UNIDAS PARA A EDUCAÇÃO, A CIÊNCIA E A CULTURA. Declaração universal dos direitos humanos. Brasília, 1998.

PINTO, Manuela A.; SILVA, Armando M. Um modelo sistémico e integral de gestão da informação nas organizações. In: CONGRESSO INTERNACIONAL 
DE GESTÃO DA TECNOLOGIA E SISTEMAS DE INFORMAÇÃO, 2., 2005, São Paulo. Anais... São Paulo: Contecsi, 2005. p. 1-24.

POORT, Joost et al. The value of Europeana: the welfare effects of better access to digital cultural heritage. Amsterdam: Seo Economic Research, 2013.

REDE MEMORIAL DE PERNAMBUCO. Carta de Recife 2.0. [2012].

REDE MEMORIAL DE PERNAMBUCO. Carta de Ribeirão Preto. 2015.

RIO DE JANEIRO. Secretaria de Estado de Cultura. Governo do Rio de Janeiro. Rede Web de Museus. Rio de Janeiro, [2017].

SANTOS NETO, Antônio Laurindo dos et al. Tecnologias de dados abertos para interligar bibliotecas, arquivos e museus: um caso machadiano.

TransInformação, Campinas, v. 25, n. 1, p. 81-87, jan./abr. 2013.

TADDEI, Roberto. Políticas públicas para acervos digitais: propostas para o Ministério da Cultura e para o setor. São Paulo: [s.n.], 2010.

TESSLER, Andrew. Economic valuation of the British Library. London: Oxford Economics, 2013.

THIRY-CHERQUES, Hermano Roberto. Pierre Bourdieu: a teoria na prática. Revista de Administração Pública, Rio de Janeiro, v. 1, n. 40, p. 27-55, jan./fev. 2006.

\title{
Network Collections: Perspectives for cultural institutions in times of digital culture
}

\begin{abstract}
In the context of economic, cultural and social transformations produced by the development of digital technologies and especially by the emergence and popularization of the Internet, the network becomes a paradigmatic object in the forms of communication and dissemination of information. Being part of this scenario, cultural institutions have produced new practices and ways of dealing with contemporary forms of storage and dissemination of cultural information from the network object as a structuring element of their doing. The present article aims to highlight the practices of production of network collections, both nationally and internationally. The analysis of the data collected, through bibliographical and documentary research, allowed us to identify existing initiatives in Brazil, as well as to map consolidated international practices in the form of services. The results of the research demonstrate that while the practices of production of network collections find technical and political barriers in the national scenario, and no national policy in effect has been identified, in the international scenario, these
\end{abstract}


practices have been disseminated and developed in North America, Europe and Oceania.

Keywords: Collections. Network. Digital culture. Cultural institutions.

Recebido em: 27/04/2017

Aceito: 05/08/2017

Anexo A - Links de acesso aos serviços culturais

\begin{tabular}{|c|c|}
\hline Serviço & Link \\
\hline APE & https://www.archivesportaleurope.net/ \\
\hline ARTstor & http://www.artstor.org/ \\
\hline British Library & http://www.bl.uk/ \\
\hline DPLA & https://dp.la/ \\
\hline DigitalNZ & https://www.digitalnz.org/ \\
\hline Europeana & http://www.europeana.eu/portal/pt \\
\hline EUScreen & http://www.euscreen.eu/ \\
\hline Galica & http://www.gallica.bnf.fr/ \\
\hline Google & https://www.google.com.br/ \\
\hline Google Art & https://www.google.com/culturalinstituite/ \\
\hline HathiTrust & https://www.hathitrust.org/ \\
\hline Internet Archive & https://archive.org/ \\
\hline JSTORE & https://www.jstor.org/ \\
\hline Rijkdmuseum & https://www.rijksmuseum.nl/ \\
\hline
\end{tabular}




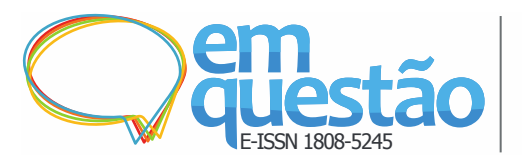

Acervos em rede: perspectivas para as instituições culturais em tempos de cultura digital

Dalton Lopes Martins, Marcel Ferrante Silva e Danielle do Carmo

\begin{tabular}{|c|c|}
\hline Smithsonian & https://www.si.edu/ \\
\hline TEL & http://www.theeuropeanlibrary.org \\
\hline Trove & http://trove.nlla.gov.au/ \\
\hline Wikimedia Commons & https://commons.wilkimedia.org/ \\
\hline Wikipedia & https://pt.wilkipedia.org/ \\
\hline World Digital Library & https://www.wdl.org/pt/ \\
\hline
\end{tabular}

${ }^{1}$ Cinemateca Brasileira e do Centro Técnico Audiovisual (CTAv), Fundação Casa de Rui Barbosa, Patrimônio Histórico e Artístico Nacional (IPHAN), Fundação Biblioteca Nacional (FBN) e Centro de Documentação da Fundação Nacional das Artes (Cedoc/Funarte).

${ }^{2}$ Casa da Marquesa de Santos / Museu da Moda Brasileira, Casa de Casimiro de Abreu, Casa de Oliveira Vianna, Casa Euclides da Cunha, Centro de Documentação da Fundação Theatro Municipal, Espaço Cultural Luciano Bastos, Museu Antônio Parreiras, Museu Bispo do Rosário de Arte Contemporânea, Museu Carmen Miranda, Museu das Telecomunicações/ Oi Futuro, Museu de História e Artes do Estado do Rio de Janeiro, Museu do Samba Carioca/ Centro Cultural Cartola e Museu Histórico da Cidade do Rio de Janeiro. 